\section{ORIGINAL RESEARCH}

B.S. Choi

J.H. Kim

C. Jung

S.Y. Kim

\title{
High-Resolution Diffusion-Weighted Imaging Increases Lesion Detectability in Patients with Transient Global Amnesia
}

BACKGROUND AND PURPOSE: DWI can detect small punctate hyperintense lesions of the hippocampus in patients with TGA. We investigated whether small TGA lesions can be detected more often by increasing the resolution of DWI.

MATERIALS AND METHODS: Of 31 consecutive patients with TGA, 27 underwent DWI, twice at the first visit (range 1.5-22 hours; mean 10 hours) and at follow-up (range 50-87 hours; mean 72.5 hours) after the onset of their symptoms. Each DWI included 2 different spatial resolutions with the same b-value (2000 seconds $/ \mathrm{mm}^{2}$ ): conventional resolution in a $128 \times 128$ matrix with $3-\mathrm{mm}$ section thickness and high resolution in a $220 \times 220$ matrix with 2 -mm section thickness. The number and contrast of hyperintense lesions were compared between the 2 resolutions.

RESULTS: Twenty-two of the 27 patients had single or multiple TGA lesions. The total number of lesions detected on conventional and high-resolution DWI was 11 and 22, respectively, at the first visit, and was 24 and 37, respectively, at follow-up. The number of lesions was significantly larger on high-resolution DWI than on conventional resolution at the first visit $(P<.01)$ and at the follow-up $(P<.01)$. Lesion contrast was significantly increased on high-resolution DWI $(P<.01)$.

CoNCLUSIONS: Higher DWI resolution increased lesion detectability in patients with TGA. Considering the small size of TGA lesions, the resolution of DWI is an important parameter influencing lesion detectability.

ABBREVIATIONS: $\mathrm{Cl}=$ confidence interval; $\mathrm{OR}=$ odds ratio; SENSE = sensitivity encoding; $\mathrm{SI}=$ signal intensity; TGA = transient global amnesia

$\mathbf{T}$ GA is a syndrome of sudden-onset anterograde and retrograde memory loss lasting for several hours, and is usually accompanied by repetitive questioning without other focal neurologic symptoms or signs. ${ }^{1}$ The incidence of TGA has been reported to be $3-8$ cases per 100,000 people per year. ${ }^{2-5}$

Many recent studies have reported that 1 - to $5-\mathrm{mm}$ punctuate high-signal intensity lesions have frequently been found in the lateral portion of the hippocampus, that is, the CA1 region, on DWI in TGA. ${ }^{6-11}$ Although imaging detection of the lesions in TGA may not change the clinical course of this self-resolving disorder or affect treatment planning, an optimal DWI protocol may help to exclude other possible urgent differential diagnoses that might clinically mimic the symptoms of TGA, such as transient ischemic attack, seizure, drug-induced amnesia, and other causes of altered awareness. ${ }^{12-15}$

The detection rate of TGA lesions on DWI has been reported as a wide range between $0 \%-84 \% .^{6,7,10,16,17}$ These variable rates of lesion detection are attributable to different imaging parameters such as section thickness, b-value, and

Received November 2, 2011; accepted after revision December 11.

From the Departments of Radiology (B.S.C., J.H.K., C.J.) and Neurology (S.Y.K.), Seoul National University College of Medicine, Seoul National University Bundang Hospital, Seongnam, Korea.

Please address correspondence to Jae Hyoung Kim, MD, Department of Radiology, Seoul National University Bundang Hospital, 166 Gumi-ro, Bundang-gu, Seongnam-si, Gyeonggi-do 463-707, Korea; e-mail: jaehkim@snu.ac.kr

Indicates article with supplemental on-line table.

http://dx.doi.org/10.3174/ajnr.A3072 imaging timing from the onset of symptoms. High b-value (ie, 2000-3000 seconds $/ \mathrm{mm}^{2}$ ), thin section thickness (ie, $3 \mathrm{~mm}$ ), and imaging 48-72 hours after the onset of symptoms increased lesion detectability of DWI. ${ }^{7,10,18}$

However, until now, there has been no well-designed study to evaluate the influence of the resolution of DWI on lesion detectability in TGA. In this study, we investigated whether punctuate TGA lesions can be detected more often by increasing the resolution of DWI.

\section{Materials and Methods \\ Patients}

Thirty-one patients were diagnosed with TGA at our institution over a period of 10 months. The criteria used to diagnose TGA were as follows: 1) the presence of anterograde amnesia, 2) witnessed by an observer, 3) no clouding of consciousness or loss of personal identity recognition, 4) cognitive impairment limited to amnesia, 5) no focal neurologic or epileptic signs, 6) no recent history of head trauma or seizures, and 7) symptom resolution within 24 hours. ${ }^{3,19}$

Twenty-seven of the 31 patients who underwent DWI twice during their first visit to the hospital, and again 2-4 days after the onset of symptoms, were included in this study. The patients consisted of 18 women and 9 men, with a mean age of 63 years (SD, 7.6 years) and a range of 42-80 years. The initial DWI was performed 1.5-22 hours (mean, 10 hours) after symptom onset and the follow-up DWI 50-87 hours (mean, 72.5 hours) after symptom onset. Informed consent was obtained from the patients or their guardians, and our institutional review board approved the study protocol.

\section{MR Imaging Sequences}

MR imaging was conducted using a 3T MR system (Intera Achieva; Philips Health Care, Best, the Netherlands) with a SENSE head coil. 
The MR imaging protocol included DWI with 2 different resolutions, T1- and T2-weighted imaging, fluid-attenuated inversion recovery imaging, conventional gradient-echo imaging in the transverse plane, T1-weighted imaging in the sagittal plane, 3D time-of-flight angiography of the intracranial region, contrastenhanced angiography of the neck region, and contrast-enhanced T1-weighted imaging. Repeated DWI was performed 2-4 days after symptom onset with the same imaging parameters. Single-shot spin-echo echo-planar imaging was used for DWI with the following parameters: TR, $5000 \mathrm{~ms}$; TE, $59 \mathrm{~ms}$; FOV, $220 \mathrm{~mm}$; matrix, $128 \times 128$; section thickness, $3 \mathrm{~mm}$ (voxel size, $1.7 \times 1.7 \times 3 \mathrm{~mm}$ in $\mathrm{x}-, \mathrm{y}-$, and $\mathrm{z}$-axis); 30 sections; SENSE factor, 2; NEX, 2; b-value, 2000 seconds $/ \mathrm{mm}^{2}$; acquisition time, 43 seconds for conventional resolution and TR, $3580 \mathrm{~ms}$; TE, $82 \mathrm{~ms}$; FOV, $220 \mathrm{~mm}$; matrix, $220 \times 220$; section thickness, $2 \mathrm{~mm}$ (voxel size, $1 \times 1 \times 2 \mathrm{~mm}$ in $\mathrm{x}-$, $\mathrm{y}$-, and $\mathrm{z}$-axis); 14 sections; SENSE factor, 2; NEX, 4; b-value, 2000 seconds $/ \mathrm{mm}^{2}$; and acquisition time 2 minutes 21 seconds for high resolution. DWI was performed in the oblique axial plane parallel to the long axis of the hippocampus.

\section{Image Analysis}

DWI of all patients was randomly ordered and analyzed. The presence or absence of hyperintense lesions was visually determined by consensus of 2 neuroradiologists. TGA lesions were defined as round or ovoid discrete hyperintense lesions at the hippocampus, with decreased ADC at the same location.

As a measure of the conspicuity of the lesion, the percent lesion contrast was calculated from the following equation:

$$
\frac{\left(\mathrm{SI}_{\text {Lesion }}-\mathrm{SI}_{\text {Adjacent Tissue }}\right)}{\mathrm{SI}_{\text {Adjacent Tissue }}} \times 100(\text { unit of } \%) .{ }^{20}
$$

$\mathrm{SI}_{\text {Lesion }}$ is the signal intensity of a single pixel that showed the highest signal intensity within the boundary of the lesion, and $\mathrm{SI}_{\text {Adjacent Tissue }}$ is the mean signal intensity of the adjacent normal-looking hippocampus by manually drawing a region of interest (size mean, $41.7 \mathrm{~mm}^{2}$, and range, $24.9-73.1 \mathrm{~mm}^{2}$ ). The measurement was performed for TGA lesions detected on both conventional resolution and high resolution at either the first visit or the follow-up. The lesions detected only on either conventional resolution or high resolution were excluded in the measurement.

\section{Statistical Analysis}

Analysis was carried out using R (http://www.R-project.org), and statistical significance was reported at the $5 \%$ level. ${ }^{21}$

We compared the number of hyperintense lesions between the 2 resolutions at the first visit and at follow-up after symptom onset, using the $\chi^{2}$ test, and examined the influence of the resolution and imaging time of DWI on lesion detectability using a generalized linear mixed model. This model considered the repeated measure of DWI and the clustering effect of multiple lesions in the same patients. The percent lesion contrast of TGA lesions was compared between the 2 resolutions with a 2 -tailed paired $t$ test.

\section{Results}

The summary of patient information and lesion detection obtained on 4 different DWIs is shown in the On-line Table. Twenty-two of the 27 patients had either a single or multiple TGA lesions, as viewed on the initial DWI and/or the follow-up DWI. Eleven patients had a single lesion, 8 had 2 lesions, 2 had 3 lesions, and 1 had 5 lesions. Therefore, a total of 38 lesions were found unilaterally in 14 patients ( 9 left, 5 right) and bilaterally in 8 patients. Fifteen of 38 lesions were detected in the right hippocampus and 23 lesions in the left hippocampus. No significant abnormal findings other than TGA lesions were detected in any patient's brain.

On initial DWI, a total of 11 lesions were detected with conventional resolution in 8 patients $(8 / 27,29 \%)$, and a total of 22 lesions were detected with high resolution in 14 patients $(14 / 27,52 \%)$. On the follow-up DWI, there were a total of 24 lesions detected with conventional resolution in 15 patients $(15 / 27,56 \%)$, and a total of 37 lesions detected with high resolution in 22 patients $(22 / 27,81 \%)$. Representative cases are shown in Figs 1 and 2.

The number of lesions detected on DWI was significantly higher on high resolution than on conventional resolution during both imaging sessions $(P<.01$ for both sessions). The number of lesions had increased significantly on the follow-up DWI compared with the first DWI for both conventional and high resolutions ( $P<.01$ for both resolutions). According to the generalized linear mixed model, both resolution and imaging timing of DWI had a strong independent association with the lesion detectability $(\mathrm{OR}=3.8 ; 95 \% \mathrm{CI}, 2.3-5.2 ; P<$ .01 for the resolution, and $\mathrm{OR}=4.3 ; 95 \% \mathrm{CI}, 2.8-5.8 ; P<.01$ for the imaging time).

The percent lesion contrast of TGA lesions was measured in 33 lesions in 22 patients. The mean and standard deviation of the percent lesion contrast were $112 \pm 52 \%$ (range 33\%$248 \%$ ) on conventional resolution DWI, and $199 \pm 88 \%$ (range 49\%-456\%) on high-resolution DWI, which were significantly different $(P<.01)$.

\section{Discussion}

DWI resolution is an important parameter influencing lesion detectability. Because TGA lesions are susceptible to the partial volume averaging effect, due to their small size (1-3 mm) and subtle diffusion restriction during the first day of symptom onset, a higher-resolution DWI seems preferable for detecting smaller lesions at the early stage. ${ }^{79}$ Prior to our study, Bartsch et $\mathrm{al}^{22}$ performed DWI at $3 \mathrm{~T}$ using the smallest voxel size $\left(1.7 \times 0.9 \times 3 \mathrm{~mm}\right.$ in $\mathrm{x}$-, $\mathrm{y}^{-}$, and $\mathrm{z}$-axis $\left.=4.7 \mathrm{~mm}^{3}\right)$, with lesion detectability of $70 \%$ within 72 hours after symptom onset. The high-resolution DWI in our study had an even smaller voxel size $\left(1 \times 1 \times 2 \mathrm{~mm}=2 \mathrm{~mm}^{3}\right)$ compared with those of previous studies $\left(4.7-14 \mathrm{~mm}^{3}\right),{ }^{9,17,22}$ and achieved lesion detectability of $52 \%$ at the first visit and $81 \% 2-4$ days after symptom onset. Because of the high SNR of the $3 \mathrm{~T} \mathrm{MR}$ system, and doubled NEX, the in-plane resolution could be increased in our study, and, therefore, high-resolution DWI at $3 \mathrm{~T}$ is recommended for patients with suspected TGA.

In our study, multiplicity of TGA lesions was found in half of the patients on high-resolution DWI. However, previous studies reported relatively lower incidence of multiplicity, ranging from $5 \%$ to $38 \% .^{7,10,22,23}$ This may be due to increased detectability of high-resolution DWI for smaller lesions, which were missed on lower resolution by partial volume averaging effects. ${ }^{7,9,22}$ Therefore, we think that high-resolution DWI with less partial volume averaging might improve lesion contrast in our study. Indeed, the percent lesion contrast of TGA lesions on high-resolution DWI was approximately doubled compared with conventional resolution. 


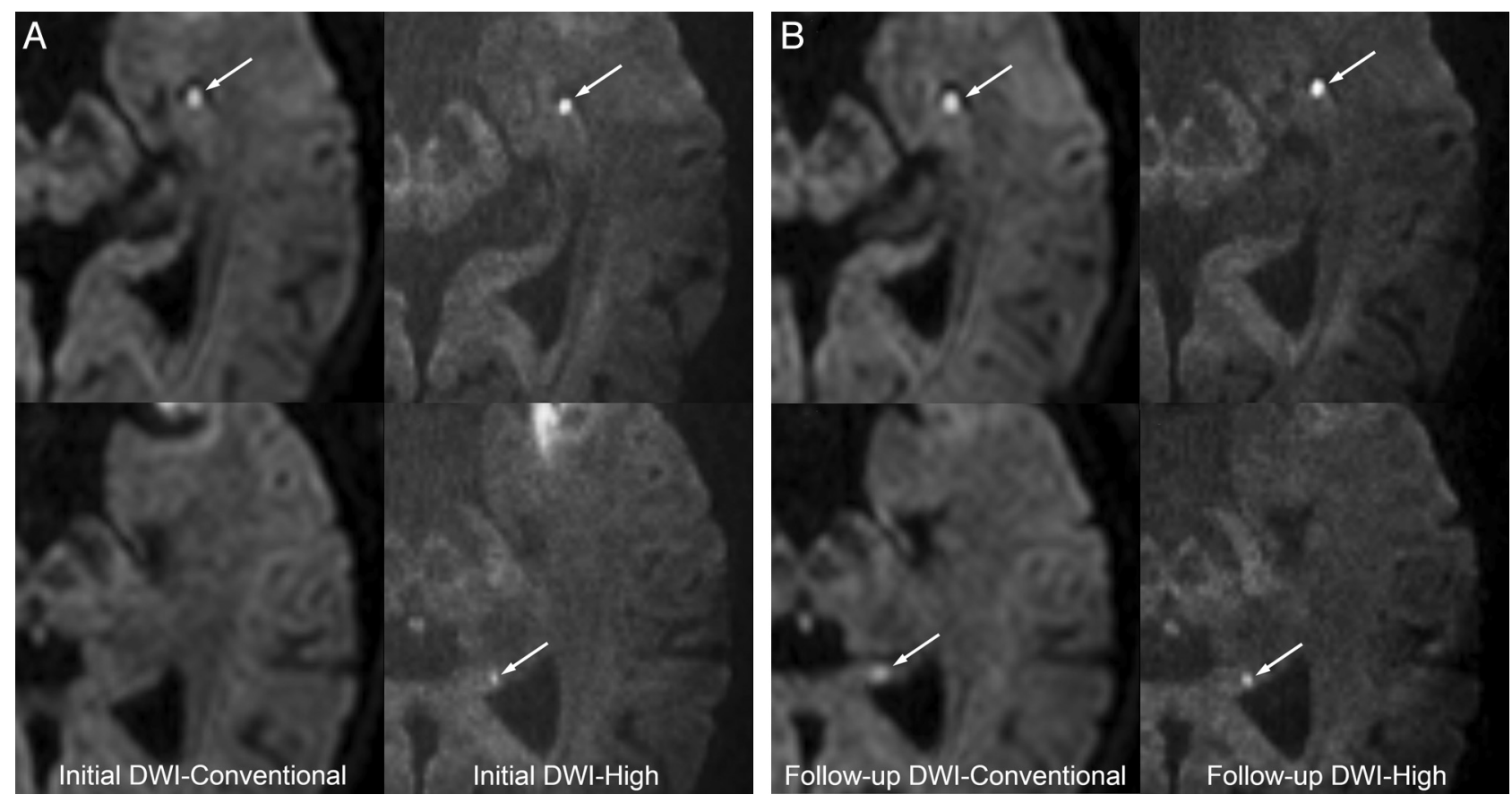

Fig 1. 72-year-old woman with TGA. A, The initial high-resolution DWI, obtained 22.5 hours after symptom onset, shows 2 hyperintense TGA lesions in the left hippocampus head and tail (arrows); however, conventional-resolution DWI shows only 1 lesion in the hippocampal head (arrow). B, On follow-up DWI performed 72 hours after symptom onset, the 2 hyperintense lesions are seen clearly with both conventional and high resolutions (arrows). Lesion contrast is better with high resolution.
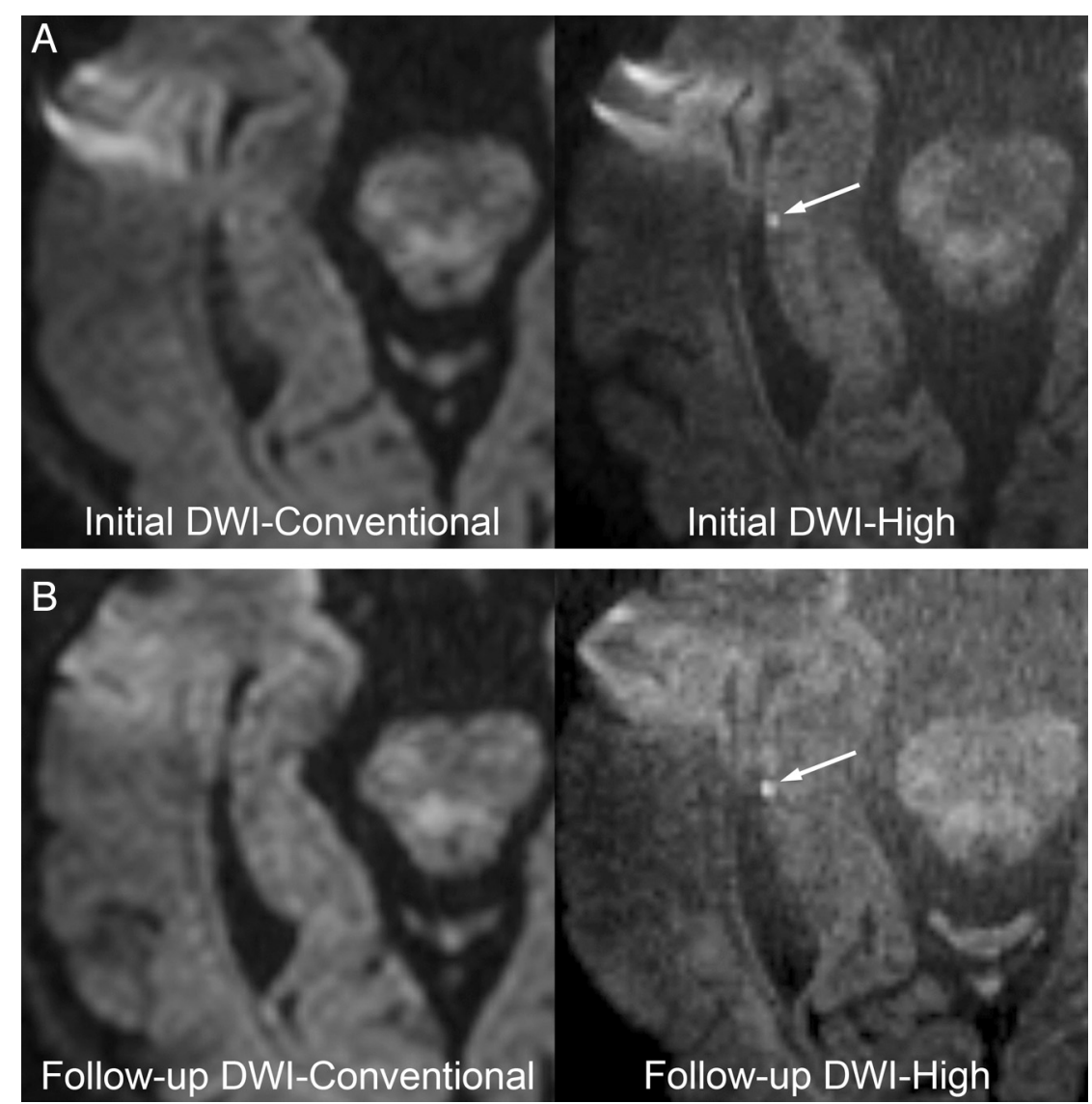

Fig 2. 66-year-old woman with TGA. $A$, The initial high-resolution DWI, obtained 12.5 hours after symptom onset, shows a hyperintense TGA lesion in the right hippocampal body (arrow). Conventional resolution DWI does not show any lesion. B, On follow-up DWI performed 85.5 hours after symptom onset, the lesion is seen only with high resolution (arrow).

A higher detection rate was achieved on DWI performed 2-4 days after symptom onset compared with the initial DWI.
This result concurred with the study by Sedlaczek et $\mathrm{al}^{7}$ in which the lesion detection rate was $6 \%$ within 8 hours of 
symptom onset and increased to $84 \% 48$ hours after symptom onset. Our study results also showed a similar effect of the time delay of DWI on lesion detectability independent from the resolution of DWI.

Similar to previous studies, ${ }^{7,22}$ TGA lesions were found slightly predominantly in the left hippocampus in our study. Although a previous study described a close relation between left posterior cerebral artery stroke and amnesia, ${ }^{24}$ the counterpoint mechanism between memory disturbance and laterality of hippocampal involvement in TGA has not been well established. Further studies are needed to investigate many unknown aspects in the pathophysiology of this curious disease.

There are some limitations in our study. As in most previous studies, the small number of the patients is a limitation. Nevertheless, statistically significant differences were obviously observed on lesion detectability and lesion contrast between the 2 resolutions. Additional studies with a larger number of the patients might be needed to investigate the pathophysiologic mechanisms of TGA. Second, there may be concerns regarding false-positive cases because of the poor SNR of high-resolution DWI. Comparative data on the SNR between the 2 resolutions could not be obtained because, due to inherent elimination of the background noise in the parallel imaging (SENSE) technique of the Philips MR system, the SNR was not accurately calculated in our MR equipment. However, higher contrast of TGA lesions and increased NEX (4 NEX in high resolution and 2 NEX in conventional resolution) could sufficiently overcome the somewhat noisy background signal.

\section{Conclusions}

High-resolution DWI significantly increased the detectability of TGA lesions. Considering the small size of TGA lesions, the resolution of DWI is an important parameter influencing lesion detectability.

\section{Acknowledgments}

The authors thank Bonnie Hami for correcting the English in this article.

\section{References}

1. Fisher CM, Adams RD. Transient global amnesia. Acta Neurol Scand 1964; 40(suppl 9):1-83
2. Miller JW, Petersen RC, Metter EJ, et al. Transient global amnesia: clinical characteristics and prognosis. Neurology 1987;37:733-37

3. Hodges JR, Warlow CP. The aetiology of transient global amnesia. A casecontrol study of 114 cases with prospective follow-up. Brain 1990;113:639-57

4. Quinette P, Guillery-Girard B, Dayan J, et al. What does transient global amnesia really mean? Review of the literature and thorough study of 142 cases. Brain 2006;129:1640-58

5. Berli R, Hutter A, Waespe W, et al. Transient global amnesia - not so rare after all. Swiss Med Wkly 2009;139:288-92

6. Strupp M, Bruning R, Wu RH, et al. Diffusion-weighted MRI in transient global amnesia: elevated signal intensity in the left mesial temporal lobe in 7 of 10 patients. Ann Neurol 1998;43:164-70

7. Sedlaczek O, Hirsch JG, Grips E, et al. Detection of delayed focal MR changes in the lateral hippocampus in transient global amnesia. Neurology 2004;62: 2165-70

8. Sander K, Sander D. New insights into transient global amnesia: recent imaging and clinical findings. Lancet Neurol 2005;4:437-44

9. Lee HY, Kim JH, Weon Y-C, et al. Diffusion-weighted imaging in transient global amnesia exposes the CA1 region of the hippocampus. Neuroradiology 2007;49:481-87

10. Weon YC, Kim JH, Lee JS, et al. Optimal diffusion-weighted imaging protocol for lesion detection in transient global amnesia. AJNR Am J Neuroradiol 2008;29:1324-28

11. Bartsch T, Deuschl G. Transient global amnesia: functional anatomy and clinical implications. Lancet Neurol 2010;9:205-14

12. Graff-Radford NR, Tranel D, Van Hoesen GW, et al. Diencephalic amnesia. Brain 1990;113:1-25

13. Kopelman MD, Panayiotopoulos CP, Lewis P. Transient epileptic amnesia differentiated from psychogenic "fugue": neuropsychological, EEG, and PET findings. J Neurol Neurosurg Psychiatry 1994;57:1002-04

14. Butler CR, Graham KS, Hodges JR, et al. The syndrome of transient epileptic amnesia. Ann Neurol 2007;61:587-98

15. Szabo K, Forster A, Jager T, et al. Hippocampal lesion patterns in acute posterior cerebral artery stroke: clinical and MRI findings. Stroke 2009;40:2042-45

16. Gass A, Gaa J, Hirsch J, et al. Lack of evidence of acute ischemic tissue change in transient global amnesia on single-shot echo-planar diffusion-weighted MRI. Stroke 1999;30:2070-72

17. Winbeck K, Etgen T, von Einsiedel HG, et al. DWI in transient global amnesia and TIA: proposal for an ischaemic origin of TGA. J Neurol Neurosurg Psychiatr 2005;76:438-41

18. Bartsch T, Alfke K, Deuschl G, et al. Evolution of hippocampal CA-1 diffusion lesions in transient global amnesia. Ann Neurol 2007;62:475-80

19. Caplan LR. Transient global amnesia. In: Vinken PJ, Bruyn GW, Klawans HL, eds. Handbook of Clinical Neurology. Amsterdam, the Netherlands: Elsevier Science Publishers, 1985:205

20. Latchaw RE. Image quality in clinical magnetic resonance imaging. In: Latchaw RE, ed. MR and CT Imaging of the Head, Neck, and Spine. 2nd ed. Boston: Mosby, 1991:3-17

21. R Development Core Team. R: A language and environment for statistical computing. Vienna, Austria: R Foundation for Statistical Computing; 2010

22. Bartsch T, Alfke K, Stingele R, et al. Selective affection of hippocampal CA-1 neurons in patients with transient global amnesia without long-term sequelae. Brain 2006;129:2874-84

23. Enzinger C, Thimary F, Kapeller P, et al. Transient global amnesia: diffusionweighted imaging lesions and cerebrovascular disease. Stroke 2008;39: 2219-25

24. Ott BR, Saver JL. Unilateral amnesic stroke. Six new cases and a review of the literature. Stroke 1993;24:1033-42 